\title{
La percepción de los estudiantes sobre su experiencia de aprendizaje a partir de su dedicación temporal y adquisición de competencias: un estudio sobre la implementación del Grado de Traducción e Interpretación de la Universidad Pompeu Fabra
}

\section{Students' perception of their learning experience from their workload and competence development: a study about the implementation of the Bachelor's degree in Translation and Interpretation of the Pompeu Fabra University}

Carles ROCA-CUBERES

Universidad Pompeu Fabra

Recibido: Octubre 2012

Aceptado: Febrero 2013

\section{Resumen}

En este estudio piloto se analiza la implantación del primer curso del nuevo Grado de Traducción e Interpretación de la Universidad Pompeu Fabra. Se examinan, mediante la encuesta y los grupos de discusión, dos parámetros: la carga de trabajo del estudiante, y la coherencia entre competencias y su implementación y evaluación. Los resultados para el primer parámetro muestran una dedicación inferior a las 25 horas por crédito ECTS deseables. Para el segundo parámetro los resultados muestran un nivel de adquisición de las competencias aceptable y suficiente. En general, a partir de los resultados obtenidos y desde un punto de vista pedagógico, se puede calificar el primer curso del Grado de Traducción e Interpretación como satisfactorio, pero con cuestiones que deben ser tomadas en consideración. A tal efecto se enumeran una serie de propuestas de mejora. Se apunta también la necesidad de llevar a cabo estudios que, como éste, examinen la percepción del estudiante sobre su experiencia educativa.

Palabras clave: EEES, competencias, ECTS, carga de trabajo, propuestas de mejora
Abstract
This pilot study analyzes the implementation of the first year of the new Bachelor's Degree in Translation and Interpretion of the Pompeu Fabra University. It examines, through a survey and focus groups, two parameters: the student's workload, and the coherence between competences and their implementation and assessment. The results for the first parameter show that the 
workload is lower than the desirable 25 hours per ECTS credit. For the second parameter the results show that competence attainment is acceptable and sufficient. In general, the first year of the Bachelor's Degree in Translation and Interpretation can be rated - from both the empirical results and a pedagogical perspective - as satisfactory. However, some issues must be addressed. For this purpose, a number of proposals for improvement are put forward. The need for studies like the present one, with a focus on students' perception of their educational experience, is also identified.

Keywords: ESHE, competences, ECTS, student workload, suggestions for improvement

Un plan de estudios tiene diferentes miradas a la hora de ser evaluado y valorado en términos de calidad. Aunque la ANECA pide principalmente indicadores numéricos, la Facultad de Traducción e Interpretación de la Universidad Pompeu Fabra ha querido examinar también de forma cualitativa en este estudio piloto en la universidad el nivel de satisfacción con el aprendizaje de competencias programado para el primer curso del nuevo Grado, a través de la percepción de las estudiantes sobre su propio aprendizaje. En esta misma línea se ha estudiado también, a través de esta investigación, si el nuevo plan de estudios basado en competencias e implementado por primera vez, está originando un aprendizaje de calidad en sus estudiantes. Por otro lado, se ha analizado la carga de trabajo de los estudiantes en términos de créditos ECTS para corroborar si ésta se equipara con los créditos planificados.

Uno de los elementos fundamentales en el diseño de un plan de estudios es la carga de trabajo a realizar por el estudiante (Kember, 2004; Mateos y Montanero, 2008). La carga de trabajo, tal y como propone Kember (2004), puede ser entendida como las horas de trabajo que dedica el estudiante a tareas tales como asistencia a clases (en grupo grande, seminarios o tutorías) o trabajo independiente fuera de las aulas (lectura de textos, trabajos en grupo, estudio para exámenes, etc.). Además, se refiere al tiempo teórico en que se puede esperar que un estudiante obtenga los resultados del aprendizaje en forma de competencias que expresen las destrezas adquiridas (EC, 2005). En el EEES se parte de la base de que 60 créditos miden la carga de trabajo de un estudiante a tiempo completo durante un curso académico, en el que un crédito representa de 25 a 30 horas de trabajo (ibid.). Lograr ajustar la carga de trabajo en función de los créditos asignados constituye una de las mayores dificultades a las que se enfrenta el profesorado (Morgan, Taylor y Gibbs, 1982; Marton, Hounsell y Entwistle, 1984; Kember, Tse, Wong y Pomfret, 1996; Kember, 2004; Zuriff, 2003; Reyes, Valdés y Castaño, 2006). De ahí, precisamente, surge la necesidad de llevar a cabo estudios como el presente $u$ otros precedentes con objetivos similares en España (Castaño, Ruiz, Gómez-Alday y De Manuel, 2006; Reyes, Valdés y Castaño, 2006).

Desde un punto de vista pedagógico la evaluación de la carga de trabajo de los estudiantes es importante puesto que se ha demostrado que una excesiva dedicación obstruye el proceso de aprendizaje (Chambers, 1992; 1994). Se ha observado, por ejemplo, que fomenta un aprendizaje superficial (Bachman y Bachman, 2006; Blumberg, 2006; Kember y Leung, 1998; Lizzio, Wilson y Simons, 2002; Ryan, Irwin, Bannon, Mulholland y Baird, 2004), lejos del deseable aprendizaje en profundidad 
comúnmente asociado a niveles de motivación positivos para el estudio (Blumberg, 2005; Parkinson, Gilling y Suddaby, 2006; Sutton, 2007). Además, una excesiva carga de trabajo se ha asociado a absentismo en las aulas (Cerrito y Levi, 1999), fracaso en los estudios (Cope y Staehr, 2005) o abandono universitario (Woodley y Parlett, 1983). En algunos casos extremos se la ha relacionado también con baja autoestima (Chambers, 1992; 1994), ansiedad y depresión (Bachman y Bachman, 2006; Diaz, Glass, Arnkoff y Tanofsky-Kraff, 2001). Desde la perspectiva de este estudio es particularmente pertinente la observación de una constante en los análisis de carga de trabajo: generalmente se ha encontrado una excesiva dedicación por parte de los estudiantes en asignaturas en las que este parámetro no había sido objeto de consideración particular (Chambers, 1992; Garg, Tuimaleali'ifano y Sharma, 1998).

La literatura científica muestra que no todos los estudiantes aprenden de la misma forma y que, en consecuencia, para alcanzar resultados de aprendizaje similares pueden necesitar tiempos de estudio sustancialmente disímiles (Chambers, 1994; Kember, Ng, Tse, Wong, y Pomfret, 1996; Marton, Hounsell y Entwistle, 1984; Morgan, Taylor y Gibbs, 1982; Zuriff, 2003). A pesar de la imposibilidad de encontrar el estudiante real 'medio', sí se ha advertido la necesidad de tomar como medida a un estudiante medio ideal, en lugar de considerar como criterio a los estudiantes extremos que puedan necesitar más o menos tiempo (Chambers, 1994).

Son muchos los factores que afectan, y en diferente grado, a la carga de trabajo de los estudiantes: por ejemplo los métodos de enseñanza, la capacidad del estudiante y los recursos pedagógicos (González y Wagenaar, 2003); la percepción del estudiante de las tareas de aprendizaje y de la carga de trabajo (Bachman y Bachman, 2006; Kember, 2004; Kember y Leung, 2006; Lockwood, 1992); habilidades de lectura y estudio (Lawless, 2000); motivación, situación personal o conocimiento previo (Chambers, 1994); dificultad, modo de evaluación, contenido y relación con el docente (Kember, 2004). Es particularmente relevante el descubrimiento de que una asignatura bien diseñada, incluso en el caso de exigencia desmesurada en términos de dedicación, no induce necesariamente en el estudiante la sensación de una excesiva carga de trabajo (Kember, 2004; Kember y Leung, 2006).

Se han utilizado diferentes métodos para evaluar la dedicación de los estudiantes. El más frecuente ha consistido en la aplicación de una encuesta en la que se demanda a los estudiantes su opinión acerca de su carga de trabajo. Bien sea a partir de una escala de likert (Bachman y Bachman, 2006; Spronken-Smith, 2005; Cope y Staehr, 2005) o con preguntas abiertas (Reisslein, Tylavsky, Matar, Seeling y Reisslein, 2007), este procedimiento ha probado su eficacia para observar la percepción de los estudiantes pero no para medir su dedicación en horas. Otro método ha implicado la distribución de formularios al final de curso de forma que los estudiantes estimaran el tiempo promedio dedicado por semana (Lawless, 2000; Zuriff, 2003), procedimiento que ha sido criticado por la poca precisión de las estimaciones que los estudiantes podían hacer a partir del recuerdo (Chambers, 1992). En otros estudios, se pidió a los estudiantes mantener un registro semanal de todas las horas dedicadas a actividades de la universidad (Cerrito y Levi, 1999; Kember, 2004). Finalmente, otro método propuesto consistió en solicitar a los estudiantes que estimaran el tiempo dedicado a 
diferentes tareas como lectura, solución de problemas, etc. (Chambers, 1992; 1994). La principal ventaja que presenta este último método es una más elevada objetividad en las estimaciones por parte de los estudiantes; por otro lado se puede hallar también un inconveniente: la dificultad de distinguir netamente entre las diferentes tareas (Garg, Vijayshre y Panda, 1992; Lawless, 2000). Como se podrá apreciar más adelante, el presente estudio ha utilizado para la estimación de la dedicación de los estudiantes una combinación de los dos últimos métodos: un procedimiento de cálculo por estimación de tareas con registro semanal.

La universidad española, inmersa en el proceso de transición al EEES (Ministerio de Educación Cultura y Deporte, 2003), ha debido adoptar un modelo de formación académica que sitúe al estudiante y su aprendizaje en el centro de la institución. Este proceso requiere un cambio tanto de mentalidad como de procedimientos por parte de los actores involucrados en su implementación (De la Cruz, 2003). En consecuencia, este modelo de formación académica ha ubicado en su base el aprendizaje por competencias en el que deben integrarse las competencias básicas, las competencias transversales que conciernen a la educación general de las personas, así como competencias específicas relacionadas con ámbitos profesionales delimitados (Martínez-Cocó et al., 2008).

El nuevo modelo de formación universitaria está suponiendo profundos cambios en las metodologías de enseñanza y evaluación. Para empezar, se fomenta una enseñanza focalizada en el aprendizaje autónomo del estudiante, que exige métodos de trabajo del profesor y del estudiante que permitan a éste último alcanzar las competencias formuladas a través de estrategias diversas (solución de problemas, aprendizaje cooperativo o por proyectos, estudio de casos, etc.). Al mismo tiempo deben diseñarse sistemas de evaluación que favorezcan y permitan valorar, a través de las estrategias mencionadas, la adquisición de competencias como expresión del aprendizaje del estudiante (Biggs, 2003; Angulo et al., 2006) mediante modalidades alternativas de evaluación (formativa, coevaluación, autoevaluación, etc.). En consonancia, se ha hecho necesaria la explicitación de no sólo los contenidos de las asignaturas, sino también del procedimiento a seguir en el desarrollo del proceso de enseñanza y evaluación, en la medida en que los métodos de trabajo sean los más adecuados para alcanzar los objetivos propuestos (García et. al, 2005), a pesar de las dificultades que se reconocen en la factibilidad de evaluar las competencias (Zabala y Arnau, 2008; Álvarez Méndez, 2008). En cualquier caso, se considera que es necesario pasar de la evaluación del aprendizaje a la evaluación para el aprendizaje y a la evaluación como aprendizaje (Gibbs y Simpson, 2004; Stiggins, 2006).

El procedimiento metodológico habitualmente usado para analizar la adquisición de competencias por parte de los estudiantes ha sido el de la encuesta. En particular, se ha utilizado en algunos estudios el Student Engagement Questionnaire para evaluar diferentes estudios de grado (Kember y Leung, 2005a; 2005b; Leung y Kember, 2005; 2006). En estas investigaciones se pide a los estudiantes que valoren su percepción sobre la adquisición de ocho competencias generales. Los resultados mostraron, por un lado, que la calidad del entorno de enseñanza y aprendizaje afecta de manera significativa al desarrollo de las competencias generales; por otro lado, durante un 
periodo de cinco años, sirvieron para llevar a cabo iniciativas de mejora de la calidad de la enseñanza. Es cada vez más notable, de todas formas, la toma en consideración de la percepción de los estudiantes sobre todos los aspectos de su experiencia en la educación superior, convertidos de esta manera en indicadores de la calidad de la universidad (Arnold et al., 1999; Hill, Lomas y MacGregor, 2003).

A continuación se presenta el método empleado en esta investigación, con los detalles de la muestra y los instrumentos de observación; se exponen los resultados obtenidos en la investigación relativos a la carga de trabajo y el desarrollo y evaluación de competencias; se proponen un conjunto de actuaciones que deben servir para mejorar el aprendizaje y la experiencia educativa de los estudiantes; finalmente, se aportan algunas conclusiones sobre el estudio realizado y se señala la necesidad de estudios similares.

\section{Método}

\section{Participantes}

En esta investigación se han estudiado dos parámetros: la carga de trabajo o dedicación temporal del estudiante, expresado en horas; la adquisición de competencias de los estudiantes. Se han analizado todas las asignaturas (19 en total; 4 en el primer trimestre, 8 en el segundo y 7 en el tercero) impartidas en el primer año de la implantación del Grado de Traducción e Interpretación en la Universidad Pompeu Fabra. En el primer curso podemos encontrar tres grandes tipos de asignaturas: de idioma, de lengua y de traducción.

Para llevar a cabo este estudio se ha utilizado una muestra de 14 estudiantes por trimestre, sobre una población total de 170 (Tabla 1). El procedimiento de muestreo ha sido aleatorio simple. La composición por sexo de la muestra ha sido de 13 mujeres y 1 hombre, y las edades han fluctuado entre los 18 y los 21 años.

Para analizar la representatividad estadística de los sujetos de la muestra se ha realizado una prueba ANOVA de diferencia de medias de las notas. En otras palabras, se han comparado, para cada una de las asignaturas analizadas en este estudio, la nota media de los sujetos de la muestra $(1,65$, en una escala de 0 a 4$)$ y la del resto de la clase $(1,61$, en una escala de 0 a 4$)$. Los resultados de la prueba $(F=0,76, p=0.71)$ muestran que no se detectan diferencias significativas (para alpha $=0.10$ ) entre la nota media obtenida por los alumnos de la muestra y aquellas obtenidas por el resto de la clase. En términos de representatividad, estos resultados indican que la muestra seleccionada resulta válida para este estudio.

\begin{tabular}{|l|c|c|}
\hline & Total estudiantes de primero & Muestra \\
\hline Dedicación temporal & 170 & 14 \\
\hline Competencias & 170 & 14 \\
\hline
\end{tabular}

Tabla 1. Población y muestra 


\section{Instrumentos}

La metodología utilizada para analizar estos parámetros (dedicación temporal y competencias) ha tomado forma a través de dos técnicas de análisis: la encuesta y el focus group (o grupo de discusión).

Para el estudio del primer parámetro se ha utilizado un cuestionario cuantitativo que se ha ido suministrando semanalmente a lo largo de cada uno de los trimestres, incluidas las fechas de examen. ${ }^{1}$ Este cuestionario tiene por objeto medir la dedicación temporal del estudiante, tanto dentro como fuera del aula, mediante un procedimiento de cálculo por estimación de tareas que los sujetos de la muestra han llevado a cabo. Los dos ítems de que consta este cuestionario permiten a los estudiantes cuantificar (semanalmente) en minutos: su asistencia a diferentes tipos de sesiones presenciales (clases en grupo grande, seminarios y tutorías); su dedicación a diferentes actividades fuera del aula (ver Tabla 5).

Para estudiar el segundo parámetro se ha utilizado un cuestionario cualitativo con preguntas abiertas que se ha suministrado tres veces coincidiendo con la finalización de cada uno de los tres trimestres. Los dos ítems de este cuestionario interrogan a los estudiantes sobre: las diferencias (en caso de haberlas) entre las competencias descritas en la guía docente de cada asignatura y las realmente desarrolladas; la medida en que han sido evaluados de esas competencias.

La otra técnica de análisis utilizada en este estudio ha sido el focus group. Con la utilización de esta técnica hemos querido corroborar los datos obtenidos con los cuestionarios respecto a los dos parámetros del estudio, pero también captar la experiencia de los estudiantes en su primer año en la universidad y detectar posibles problemas de carácter pedagógico y de adaptación. El uso de grupos de discusión se ha considerado metodológicamente adecuado para este propósito de investigación. Es una técnica que permite a los estudiantes sentirse más cómodos para expresar sus opiniones puesto que se encuentran en la compañía de amigos y compañeros. Al mismo tiempo, estos grupos proporcionan un foro en el que pueden reflexionar y reaccionar a las opiniones de otros. Además, tal y como señala Parahoo (1999), los grupos de discusión pueden poner al descubierto conflictos y desacuerdos subyacentes, desconocidos por el investigador o miembros del grupo.

Para una adecuada aplicación de la técnica del focus group se celebraron dos sesiones. Cada una de las sesiones ha sido grabada en vídeo y posteriormente se ha procedido a la transcripción de los datos. Para cada una de las sesiones se ha convocado a un mínimo de seis participantes: 7 en la primera sesión y 7 en la segunda.

\footnotetext{
${ }^{1}$ Se ha decidido contabilizar las 12 semanas del trimestre (incluyendo las 2 últimas de exámenes) y no sólo las 10 lectivas por diversos motivos. En primer lugar, si los cálculos se limitan a las 10 semanas lectivas, la dedicación por crédito ECTS es sustancialmente inferior (unas dos horas menos). Esto es así porque, por ejemplo, los estudiantes tienden a concentrar la actividad fuera del aula 'estudiar para exámenes' en esas dos últimas semanas en que no hay actividades lectivas. Esta es una actividad que, por volumen de dedicación (ver Tabla 5), no se puede desestimar. Además, las comparaciones entre asignaturas estarían sesgadas en función de si tienen o no examen final.
} 


\section{Resultados}

\section{Carga de trabajo}

El tiempo total medio dedicado por alumno en sus estudios es de 17 horas y 21 minutos por cada crédito ECTS, cantidad sustancialmente inferior a lo estipulado en el Plan de Estudios ( 1 crédito ECTS $=25$ horas). Esta cantidad (D) se ha calculado dividiendo el número total de horas de dedicación por alumno $\left(\mathrm{H}_{\mathrm{i}}\right)$ entre su carga lectiva en créditos ECTS $\left(\mathrm{C}_{\mathrm{i}}\right)$, y posteriormente tomando la media entre los N alumnos participantes en el estudio:

$$
D=\frac{1}{N} \sum_{i=1}^{N} \frac{H_{i}}{C_{i}}
$$

En la evolución por trimestres, se detecta una clara tendencia descendente en la carga de trabajo por crédito: de las 19 horas y 47 minutos por crédito del primer trimestre, se pasa a poco más de 14 horas en el último trimestre (Tabla 2). Esta disminución puede ser interpretada como resultado del proceso de adaptación de los alumnos a los estudios universitarios (cf. Sutton, 2007) y también del diferente perfil de las asignaturas cursadas en cada trimestre.

\begin{tabular}{|l|l|l|}
\hline Trimestre & Minutos & Horas y minutos \\
\hline Primero & 1187 & 19 horas y 47 minutos \\
\hline Segundo & 1085 & 18 horas y 5 minutos \\
\hline Tercero & 848 & 14 horas y 8 minutos \\
\hline MEDIA & $\mathbf{1 0 4 1}$ & $\mathbf{1 7}$ horas y 21 minutos \\
\hline
\end{tabular}

Tabla 2. Tiempo total dedicado por alumno, trimestre y crédito ECTS

Nota: Incluye las 12 semanas del trimestre (10 semanas lectivas y 2 semanas de exámenes). 1 crédito ECTS $=25$ horas

En cuanto a la distribución de horas lectivas y no lectivas (Tabla 3), la dedicación temporal del alumno fuera del aula representa alrededor del $69 \%$ del tiempo total que dedica a cada asignatura. Se puede observar cómo los dos tipos de dedicación a los estudios disminuyen su importancia, en tiempo, según avanzan los trimestres. Este descenso es particularmente intenso en el caso de las horas fuera del aula, que se reducen a la mitad del primer al tercer trimestre. Esto hace que, en términos porcentuales, las horas dentro del aula representen una proporción mayor de la dedicación a los estudios en el tercer trimestre, aunque en términos absolutos la dedicación sea menor. Como en el caso anterior, esta tendencia podría estar relacionada con una mejor adaptación de los alumnos al ritmo de trabajo de la universidad (cf. Sutton, 2007) y con el tipo de asignaturas de cada trimestre. 


\begin{tabular}{|l|l|l|l|l|}
\hline Trimestre & Dentro del aula & $\mathbf{\%}$ & Fuera del aula & $\mathbf{\%}$ \\
\hline Primero & 127 & $27 \%$ & 342 & $73 \%$ \\
\hline Segundo & 114 & $31 \%$ & 258 & $69 \%$ \\
\hline Tercero & 109 & $39 \%$ & 174 & $61 \%$ \\
\hline MEDIA & $\mathbf{1 1 7}$ & $\mathbf{3 1 \%}$ & $\mathbf{2 5 7}$ & $\mathbf{6 9 \%}$ \\
\hline
\end{tabular}

Tabla 3. Proporción de tiempo de dedicación a los estudios, dentro y fuera del aula, por alumno, semana y trimestre

Nota: Incluye las 12 semanas de cada trimestre (10 semanas lectivas y 2 semanas de exámenes). Los tiempos dentro y fuera del aula están expresados en minutos por semana, y representan el promedio por estudiante.

Al profundizar en la distribución de las horas dentro del aula (Tabla 4), los datos muestran como, de manera aproximada, se cumplen las previsiones de los planes de estudio (65\% de clases magistrales, 35\% de seminarios y tutorías). La distribución es de $63 \%, 36 \%$ y $1 \%$. La única excepción en este sentido es la relativa a las tutorías con los profesores, que representan un porcentaje casi nulo sobre el total de tiempo dedicado a los estudios. Los resultados son consistentes a lo largo del curso, con sólo una pequeña disminución en el tiempo total de horas lectivas, en términos absolutos, que podría estar relacionada con una mayor tasa de absentismo (tal y como observan Cerrito y Levi, 1999) o una diferente planificación de las asignaturas.

\begin{tabular}{|l|l|l|l|l|l|l|}
\hline Trimestre & $\begin{array}{l}\text { Grupo } \\
\text { grande }\end{array}$ & $\mathbf{\%}$ & Seminarios & $\mathbf{\%}$ & Tutorías & $\%$ \\
\hline Primero & 99 & $65 \%$ & 52 & $34 \%$ & 1 & $1 \%$ \\
\hline Segundo & 81 & $62 \%$ & 49 & $37 \%$ & 1 & $1 \%$ \\
\hline Tercero & 79 & $61 \%$ & 48 & $38 \%$ & 1 & $1 \%$ \\
\hline MEDIA & $\mathbf{8 6}$ & $\mathbf{6 3 \%}$ & $\mathbf{5 0}$ & $\mathbf{3 6 \%}$ & $\mathbf{1}$ & $\mathbf{1 \%}$ \\
\hline
\end{tabular}

Tabla 4. Distribución de la dedicación dentro del aula

Nota: Incluye únicamente las 10 semanas lectivas de los trimestres. Los tiempos están expresados en minutos (media semanal de minutos dedicados a cada tipo de actividad dentro del aula).

En relación a las horas fuera del aula se observan también diferencias en el peso que cada tipo de actividad representa sobre la dedicación de los alumnos en sus estudios (Tabla 5). Se comprueba, por ejemplo, como gran parte de la reducción de esta dedicación se debe a una menor realización de ejercicios de redacción y trabajos en grupo, menor frecuencia en lectura de artículos, textos y libros, y una inferior proporción de tiempo orientado al estudio para los exámenes. El resto de actividades mantienen un peso sobre la dedicación total a los estudios que es relativamente constante a lo largo de los trimestres, con las variaciones normales, en relación a los diferentes tipos de asignaturas de cada uno de ellos. 


\begin{tabular}{|l|l|l|l|l|}
\hline & \multicolumn{3}{|l|}{ TRIMESTRE } & \multicolumn{1}{l}{} \\
\hline ACTIVIDADES & $\mathbf{1}$ & $\mathbf{2}$ & $\mathbf{3}$ & MEDIA \\
\hline Ejercicios de redacción & 64 & 48 & 30 & 47 \\
\hline Lecturas de artículos, textos, libros & 49 & 45 & 21 & 38 \\
\hline Comentarios de texto & 2 & 4 & 0 & 2 \\
\hline Ejercicios de lengua & 16 & 23 & 25 & 22 \\
\hline Ejercicios de traducción & 7 & 24 & 11 & 15 \\
\hline Impresión de documentos & $\mathrm{n} / \mathrm{d}$ & 3 & 2 & 3 \\
\hline Revisión de textos ya corregidos & 9 & 6 & 3 & 6 \\
\hline Pasar los apuntes a limpio & 13 & 8 & 10 & 10 \\
\hline Hacer resúmenes o esquemas & 18 & 11 & 7 & 12 \\
\hline Buscar información en internet & 19 & 4 & 10 & 11 \\
\hline Buscar información en bibl. recomendada & 11 & 1 & 1 & 4 \\
\hline Estudiar para exámenes & 53 & 39 & 33 & 42 \\
\hline Preparar exposiciones orales individuales & 5 & 0 & 1 & 2 \\
\hline Encontrarme con mi grupo de trabajo & 35 & 33 & 4 & 24 \\
\hline Consultar documentos audiovisuales & 2 & 2 & 4 & 2 \\
\hline Otros & 36 & 7 & 13 & 18 \\
\hline
\end{tabular}

Tabla 5. Distribución de la dedicación fuera del aula

Nota: Incluye las 12 semanas de cada trimestre (10 semanas lectivas y 2 semanas de exámenes). Los tiempos están expresados en minutos (media semanal de minutos dedicados a cada tipo de actividad fuera del aula). Los datos para la tarea 'de impresión de documentos' del primer trimestre no están disponibles.

Buena parte de las observaciones realizadas anteriormente se pueden contrastar de manera visual en el Gráfico 1. Se aprecia, en primer lugar, la notable reducción a lo largo de los trimestres que se detecta en el tiempo que los alumnos dedican a sus estudios fuera del aula, mientras que las horas dentro del aula se mantienen relativamente constantes o con ligeras variaciones. La conclusión más relevante que podemos obtener de este gráfico es la diferente evolución de la carga de trabajo fuera del aula a lo largo de cada trimestre: (1) en el primer trimestre se aprecia una clara tendencia ascendente, con una fuerte concentración del trabajo en las últimas semanas, lo cual indica ciertos problemas de adaptación de los alumnos al ritmo de trabajo universitario (cf. Sutton, 2007) en el nuevo Plan de Estudios. (2) Esta desigualdad en la distribución del trabajo disminuye en el segundo trimestre. (3) En el tercer trimestre, a pesar de una más intensa dedicación al estudio en las últimas semanas, la carga de trabajo que suponen las asignaturas está mucho más constantemente distribuida. 


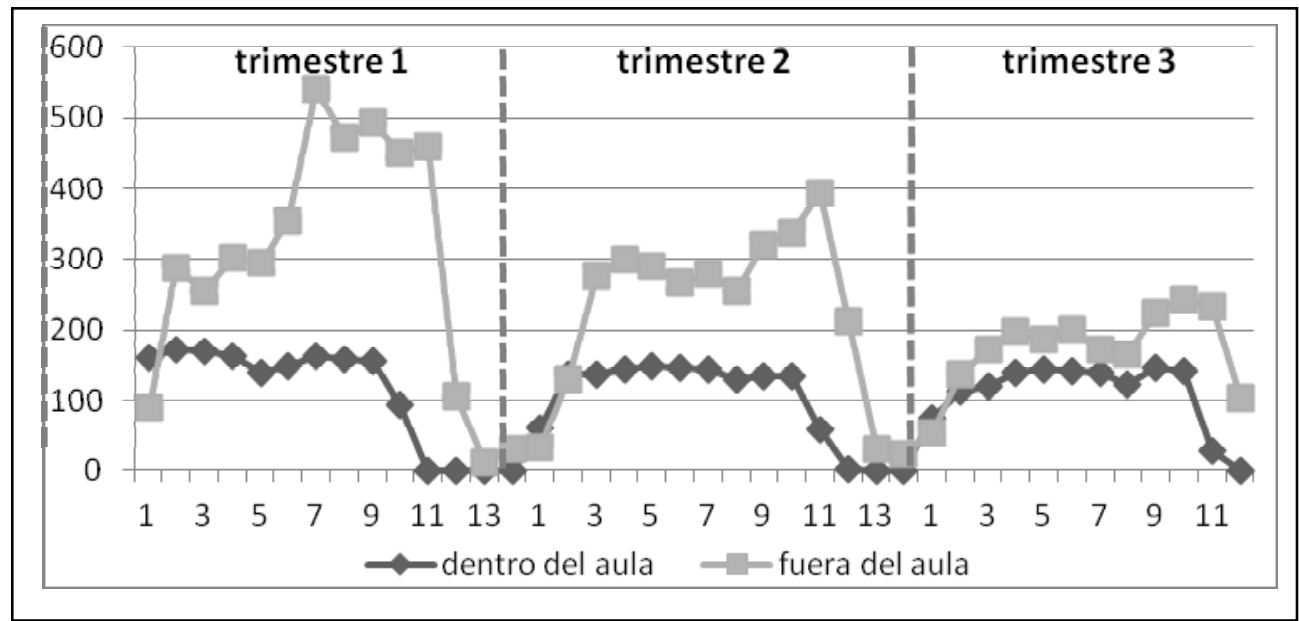

Gráfico 1. Evolución del tiempo semanal dedicado por alumno y asignatura dentro y fuera del aula. El eje horizontal indica las semanas desde el inicio de cada trimestre. El eje vertical muestra la dedicación por estudiante, en minutos por semana.

Los resultados de los grupos de discusión, celebrados a final de curso, corroboran respecto a este parámetro lo observado a lo largo de los tres trimestres: la dedicación de los estudiantes en horas por crédito ECTS ha seguido una línea descendente (primer trimestre: 19 horas y 29 minutos; segundo trimestre: 18 horas y 18 minutos; tercer trimestre: 14 horas y 8 minutos). En palabras de los propios estudiantes se aprecia que "el problema estuvo en el primer trimestre, porque nos lo encontramos de golpe, y es en el que más trabajo hemos tenido, junto con el segundo; y, claro, te tienes que acostumbrar; a mí el segundo me fue mucho mejor, a la hora de organizarme, pero en el primero íbamos desorientados", o "que el trabajo ha ido bajando mucho, el primer trimestre fue muy, muy costoso, y este último es que no hemos tenido casi nada de trabajo, hemos trabajado muy pocas horas". Esta dedicación descendente por crédito parece tener dos explicaciones: por un lado, la carga de trabajo requerida por las asignaturas de los diferentes trimestres ha ido disminuyendo (especialmente en el tercer trimestre); por otra, parece que los estudiantes han aprendido a maximizar, racionalizar y mecanizar las tareas de su trabajo: "hemos aprendido a organizarnos, porque yo creo que es muy diferente como hice el primer trimestre que el segundo y el tercero; he llevado mejor el trabajo". Esta apreciación indica que, con la experiencia de adaptación, las diferencias de aprendizaje entre estudiantes tienden a minimizarse (vid. Zuriff, 2003).

Muchos de ellos apuntan a un problema común: el exceso de trabajos en grupo y su dificultad para poder encontrarse: "tal vez, en el segundo trimestre, cuando teníamos cada semana trabajos en grupo, es un poco difícil quedar, sobre todo si alguien trabaja ya, o tiene clase". Este problema se ve amplificado cuando el número de estudiantes 
por grupo es excesivo ("sí, porque en algunos trabajos éramos seis o siete, y claro, cada uno tenía sus horarios, y no coincidíamos en horas libres casi nadie") o cuando una amplia oferta de asignaturas (con la consecuente variedad de horarios) limita su disponibilidad para coincidir ("el primer trimestre aún, porque coincidíamos más o menos, sólo cambiaba algún seminario, pero luego como unos tienen Francés, nosotros Alemán, cuando nosotros teníamos tal vez una hora y media libre, tal vez los de Francés tenían una hora de seminario, y no podíamos quedar"). Un buen diseño de las asignaturas, tanto en lo relativo a evaluación (Kember, 2004) como a actividades de aprendizaje (Bachman y Bachman, 2006; Blumberg, 2005; Lizio, Wilson y Simons, 2002) debería contribuir a evitar problemas de esta índole.

La cuestión de la idoneidad en la distribución de las horas lectivas (clases en grupo grande, seminarios y tutorías; idealmente, 65\%-30\%-5\%, respectivamente) parece suscitar bastante cuórum: consideran los seminarios como fundamentales para su aprendizaje: "yo creo que con lo que más hemos aprendido es con los trabajos; yo si no hubiéramos hecho todos los trabajos que hemos hecho, la mitad de las cosas no las hubiera aprendido, porque sino todo se queda en el aire, los seminarios son muy útiles". Todos asienten cuando se les pregunta por el tipo de sesión que más necesitaron: para ellos fue, claramente, el seminario. Las clases en grupo grande, eventualmente, también se consideran necesarias ("porque hay mucha teoría para diez clases, es mucho; si hubiera más clases, se podría ir con más calma, y explicarlo mejor").

Como se ha podido observar a lo largo de los trimestres, las tutorías han tenido un papel insignificante (menos de 1\%) dentro de las horas lectivas. A partir de las respuestas de los estudiantes se puede deducir que un incremento en el número de estas les habría beneficiado ("yo he hecho bastantes, y estoy contenta").

La distribución de la carga de trabajo de los estudiantes a lo largo del trimestre es considerada, a grandes rasgos, como adecuada ("en general, he tenido mucho menos trabajo [en el segundo y tercer trimestre], quizás también somos nosotros, que nos hemos adaptado al sistema"). De todas formas entienden que esta distribución está muy condicionada por la propia organización del profesor. Así, "creo que los profesores también nos lo han puesto más fácil en el segundo trimestre; en el primer trimestre todo, por casualidad o no, caía en la misma semana, de golpe; hubo dos semanas que había que entregar todo; en cambio, los otros, muchos profesores ya hacían los planes semanales, e iba distribuido diferente por semana, es decir, aparte de nosotros, también era por los profesores". Dicha organización deficiente, como Chambers (1992; 1994) argumenta, puede suponer un exceso de trabajo que obstruya el proceso de aprendizaje.

\section{Desarrollo de competencias y evaluación}

El cuestionario cualitativo 'coherencia entre competencias y su implementación y evaluación en el Grado de Traducción e Interpretación' (pasado al final de cada trimestre) tenía como objetivo examinar el grado de adquisición de las competencias por parte de los estudiantes, según la percepción que transmitían basándose en las guías 
docentes de que disponían. Los resultados agregados de los tres trimestres muestran un nivel de adquisición aceptable y, en buena medida, suficiente.

El análisis exhaustivo de las respuestas de los estudiantes en el cuestionario cualitativo sobre la correspondencia entre las competencias planteadas en los planes docentes de las asignaturas y las que consideran que han aprendido, revela que el nivel de aceptación (a pesar de las variaciones entre asignaturas) es correcto. En cuanto a la evaluación de las competencias se llega, en general, a un nivel más amplio de satisfacción.

Los datos obtenidos a partir de los grupos de discusión para este segundo parámetro se encuentran en consonancia con los resultados que hemos ido encontrando a lo largo de los tres trimestres. Así, por ejemplo, al ser preguntados sobre su nivel de aprendizaje de las competencias, se observan ciertas reticencias: "te ponen un listado bastante extenso, no puedes alcanzar todo esto en tres meses, es imposible"; "hay muchas cosas que deberíamos haber hecho, y que yo tenía la sensación de no haber hecho, el trabajo en grupo o el trabajo individual, que lo ponía en todas partes, sí, pero otros...". Obviamente, los estudiantes trasladan la cuestión al terreno de su propia experiencia y recuerdo, que en buena medida se estructura en forma de asignaturas más que de manera abstracta: "depende de la asignatura", o "en una asignatura, por ejemplo, que hicimos un portafolio y un informe final donde hablaba de cada competencia, y teníamos una tutoría al final de trimestre, sí lo he aprendido, pero en las otras no sé".

La más amplia satisfacción en cuanto a la evaluación de las competencias sí se ve reflejada en las observaciones de los estudiantes: "yo creo que sí, sobre todo en una asignatura, que el profesor que teníamos el trimestre pasado, nos hacía muchos tipos de evaluación: test, examen, redacciones, incluso la parte oral quedaba en parte evaluada; hacíamos ejercicios dirigidos a evaluar nuestro progreso, nuestras competencias". Esta observación apunta en la dirección indicada por Biggs (2003) o Angulo et al. (2006), según la cual la variedad y el uso de modalidades alternativas de evaluación favorece el aprendizaje. Además, el buen diseño de las asignaturas no produce sensación de sobrecarga y también auspicia un buen aprendizaje (Kember, 2004; Kember y Leung, 2006): "teníamos mucho trabajo, pero aprendíamos más, y quedaba todo cubierto".

Los estudiantes aprovechan sus comentarios sobre evaluación para poner de manifiesto su descontento con los métodos que se emplean ("yo creo que haces mucho trabajo y luego no se valora; has dedicado mucho de tiempo y después realmente está valorada en un porcentaje que no es lo que correspondería") y de manera especial, los exámenes ("yo es que con los exámenes no he aprendido, me han servido como para hacer un resumen de lo que he aprendido, pero no implica nada nuevo; como herramienta de aprendizaje no, por eso creo que está muy bien que no cuenten tanto los exámenes"; "yo, en general, creo que con las prácticas hemos aprendido más"). Parece claro que, como apuntan Gibbs y Simpson (2004), se hace necesario el empleo de instrumentos de evaluación que sustenten el aprendizaje del alumnado, particularmente a través de su motivación (Stiggins, 2006). 
A partir de los datos obtenidos para el segundo parámetro se han identificado problemas de diversa índole - recordando, nuevamente, que la información recogida para identificar los problemas proviene de los estudiantes - que, de forma sintética, se exponen a continuación.

- Los alumnos sienten (en el primer trimestre) que su adaptación al sistema universitario no es del todo satisfactoria.

- Se producen algunas confusiones conceptuales (especialmente en el primer trimestre) que deberían resolverse: evaluación equivale a nota, o temas (contenido) son equivalentes a competencias.

- Las competencias (sobre todo las generales) expresan un exceso de vaguedad y abstracción que dificulta su comprensión. Esto puede obstruir su efectivo traslado a la evaluación.

- Algunas competencias que se describen en las guías docentes no se trabajan suficientemente, o nada. Pueden constituir una mera declaración de intenciones o tener una finalidad estética.

- Se incluyen algunas competencias que se escapan del ámbito de alguna asignatura determinada.

- La metodología de aprendizaje y enseñanza puede no ser (en ocasiones) la correcta para facilitar el aprendizaje de las competencias.

\section{Propuestas de mejora}

En esta investigación se constata que en el primer curso del nuevo Grado del Departamento de Traducción e Interpretación se han alcanzado una serie de objetivos. Así, se ha detectado un nivel amplio de satisfacción con el curso y el proceso y resultados del aprendizaje. No obstante, algunos aspectos que quedan recogidos en las siguientes propuestas de mejora, podrían ser objeto de actuación. Estas propuestas de mejora están dirigidas a tres ámbitos de actuación: la dedicación temporal del estudiante, la adquisición y evaluación de competencias, y la adaptación del estudiante al EEES.

\section{Propuestas de mejora: dedicación temporal del estudiante}

Ajuste de los créditos ECTS al tiempo de estudio real de los estudiantes

- Ajustar la carga de trabajo de cada asignatura en función de su número de créditos ECTS (Marton, Hounsell y Entwistle, 1984).

- Distribuir la carga de trabajo de los estudiantes, a lo largo de los trimestres del primer curso, en función de su ritmo de adaptación y madurez (Morgan, Taylor y Gibbs, 1982).

\section{Acción docente}

- Coordinar los picos de trabajo de los estudiantes entre las asignaturas de un mismo trimestre. 
- Coordinar la docencia, teniendo en cuenta la distribución de los créditos ECTS y el tiempo de dedicación de los estudiantes para cada asignatura (Castaño et al., 2006).

- Distribuir el tiempo de estudio del estudiante de manera más constante y equilibrada durante el trimestre (Kember et al., 1996).

Propuestas de mejora: adquisición y evaluación de competencias

\section{Competencias}

- Afinar la correspondencia entre el planteamiento de las competencias de las asignaturas y su implementación y evaluación (González y Wagenaar, 2003; Álvarez Méndez, 2008; Zabala y Arnau, 2008).

- Incluir en la guía docente sólo aquellas competencias que los estudiantes deben desarrollar. Debería haber una clara conexión entre el objetivo declarado de la asignatura y lo que aprenden los estudiantes (González y Wagenaar, 2003; Álvarez Méndez, 2008; Zabala y Arnau, 2008).

\section{Metodología de aprendizaje y enseñanza}

- Ajustar la metodología de aprendizaje y enseñanza a las competencias de la asignatura y no al revés (De la Cruz, 2003).

- Revisar, a pesar de la satisfacción general de los estudiantes con la cantidad de seminarios, la adecuación de la relación teoría-práctica de algunas asignaturas.

- Reflexionar sobre la idoneidad del uso de los trabajos en grupo como instrumento de aprendizaje y evaluación.

- Suministrar 'feedback' a los estudiantes (sólo en los casos en que no se produzca) sobre las tareas que realizan de la forma más profunda e inmediata posible (Stiggins, 2006).

\section{Evaluación}

- Definir y explicar de forma clara los instrumentos (y sus medidas) destinados a la evaluación (García et al., 2005).

- Evaluar todas las competencias descritas en la guía docente (González y Wagenaar, 2003).

- Reflexionar sobre la idoneidad de los exámenes como instrumento de evaluación y (en caso de mantener su uso) sobre de su peso en el conjunto de la evaluación (Gibbs y Simpson, 2004; Stiggins, 2006; Martínez-Cocó et al., 2008).

\section{Propuestas de mejora: adaptación del estudiante al EEES}

- Elaborar una web para la acogida de los estudiantes de primero, con información sobre el Grado, opiniones de otros estudiantes, etc.

- Potenciar las competencias de la asignatura 'Introducción a la Universidad'. 
- Hacer un seguimiento y tutorización de los estudiantes de primero con dificultades de aprendizaje (Sutton, 2007).

- Garantizar que todas las asignaturas tengan una guía docente clara y con calendario detallado (Mateos y Montanero, 2008).

\section{Conclusiones}

Este estudio demuestra la necesidad de evaluar la adecuación de los planes de estudio creados a partir de la implantación de los nuevos Grados surgidos al calor de la transición de la enseñanza superior hacia el EEES. En la medida en que el EEES sitúa al estudiante en el centro del proceso educativo, se corrobora la necesidad de explorar la percepción que éste tiene sobre su experiencia educativa en la universidad (vid. Arnold et al., 1999; Hill, Lomas y MacGregor, 2003) sin menoscabo del uso de otros indicadores para alcanzar el mismo objetivo. Los resultados de esta exploración, como se ha observado anteriormente, constituyen indicadores que son expresión de calidad institucional y, si cabe aún más importante, de la calidad del aprendizaje del alumnado (Biggs, 2003). La satisfacción del estudiante con su proceso educativo apunta, en definitiva, a una elevada correlación con su propio aprendizaje.

Este estudio, piloto en la Universidad Pompeu Fabra (y que se ha tomado como modelo en el resto de facultades de esta universidad, en las que se están llevando a cabo las mismas investigaciones), ha examinado el nivel de adecuación del Grado de Traducción e Interpretación en su primer año de impartición a partir de dos parámetros: (1) la congruencia de las previsiones en ECTS de las asignaturas con la dedicación real de los estudiantes; (2) la coherencia entre la previsión de las competencias a desarrollar en las asignaturas, y su implementación y evaluación real.

Los resultados de esta investigación indican que las competencias fueron adquiridas de manera aceptable y suficiente, y que la dedicación temporal fue insuficiente para alcanzar los créditos ECTS planificados. Dado que el objetivo final de este estudio no se limita al análisis sino que también busca la mejora, se han concebido una serie de actuaciones que deben servir para enmendar esta situación. Entre las propuestas para mejorar la dedicación temporal del estudiante destacan, fundamentalmente, la necesidad de ajustar la carga de trabajo de cada asignatura a sus créditos ECTS, así como una distribución más equitativa de esta carga a lo largo de cada trimestre. Una mejor coordinación docente debería contribuir, sin duda, a mejorar este último aspecto. En cuanto a las posibles actuaciones para mejorar la adquisición y evaluación de competencias se debe afinar la correspondencia entre el planteamiento de las competencias y su implementación y evaluación, concibiendo las competencias como punto de partida para el desarrollo de la metodología de aprendizaje y enseñanza de cada asignatura. Los estudiantes de primero deben ser objeto de atención especial por parte de la universidad en el EEES, lo que debe conducir a un suministro exhaustivo de información sobre el Grado que empiezan a cursar; para aquellos estudiantes cuyo aprendizaje sea problemático se deberían considerar soluciones como la tutorización. 
Esta investigación ha abierto la senda a estudios similares dentro de la Universidad Pompeu Fabra, pero también puede servir de inspiración para estudios de otras universidades que deseen evaluar la calidad y adecuación de sus Grados desde la perspectiva de la experiencia educativa de sus estudiantes.

\section{Referencias bibliográficas}

ÁLVAREZ MÉNDEZ, J. M. (2008). Evaluar el aprendizaje en una enseñanza centrada en competencias. En J. Gimeno (Ed.), Educar por competencias ¿qué hay de nuevo? (pp.206-233). Madrid: Morata.

ANGULO, J., CORPAS, C., GARCÍA, J.D., GONZÁLEZ, I., Y MÉRIDA, R. (2006). Las competencias de la titulación de psicopedagogía a nivel andaluz: investigando la opinión del profesorado, del alumnado universitario y de los profesionales de la orientación. Revista de Investigación Educativa, 24(2), 575-594.

ARNOLD, J., LOGAN-CLARKE, J., HARRINGTON, A., Y HART, C. (1999). Students'perceptions of competence development in undergraduate business-related degrees. Studies in Higher Education, 24(1), 43-59.

BACHMAN, L. Y BACHMAN, C. (2006). Student perceptions of academic workload in Architectural education. Journal of Architectural and Planning Research, 23(4), 271-304.

BIGGS, J. (2003). Teaching for quality learning at university. Buckingham: The Society for Research into Higher Education and Open University Press.

BLUMBERG, P. (2005). Why self-directed learning is not learned and practiced in veterinary education. Journal of Veterinary Medical Education, 32(3), 290-295.

CASTAÑO, S., RUIZ, J. R., GÓMEZ-Alday, J. J. y De Manuel, T. (2006). Adaptación metodológica al EEES: resultados de una experiencia. Revista de la Red-U, 5(1), $35-48$.

CERRITO, P. B., y LEVI, I. (1999). An investigation of student habits in mathematics courses. College Student Journal, 33(4), 584-588.

CHAMBERS, E. (1992). Workload and the quality of student learning. Studies in Higher Education, 17(2), 141-153.

CHAMBERS, E. (1994). Assessing learner workload. En F. Lockwood (Ed.), Materials production in open and distance learning (pp. 103-111). London: Paul Chapman Publishing.

COPE, C., y STAEHR, L. (2005). Improving students' learning approaches through intervention in an information systems learning environment. Studies in Higher Education, 30(2), 181-197.

DE LA CRUZ, M. A. (2003). Necesidades y objetivos de la formación pedagógica del profesorado universitario. Revista de Educación, 331, 35-66. 
DIAZ, R. J., GLASS, C. R., ARNKOFF, D. B., Y TANOFSKY-KRAFF, M. (2001). Cognition, anxiety, and prediction of performance in 1st-year law students. Journal of Educational Psychology, 93(2), 420-429.

EC (EUROPEAN COMMISSION) (2005). ECTS Users' guide. European credit transfer and accumulation system and the diploma supplement. European Commission - Directorate General for Education and Culture.

GARCÍA, J. N., DE CASO, A. M., FIDALGO, R., Y ARIAS-GUNDÍN, O. (2005). La evaluación de prácticas universitarias y su aplicación en un enfoque innovador. Revista de Educación, 337, 295-325.

GARG, S., TUIMALEALI'IFANO, E., Y SHARMA, S. C. (1998). A study of student workload for USP physics foundation courses offered at a distance. Indian Journal of Open Learning, 7(3), 301-311.

GARG, S., VIJAYSHRE, Y PANDA, S. (1992). A preliminary study of student workload for IGNOU physics elective courses. Indian Journal of Open Learning, $1(2), 19-25$.

GIBBS, G. Y SIMPSON, C. (2004). Does your assessment support your students' learning? Journal of Teaching and Learning in Higher Education, 1, 3-32.

GONZÁLEZ, J., Y WAGENAAR, R. (2003). Tunning educational structures in Europe. Bilbao: Universidad de Deusto.

HILL, Y., LOMAS, L. Y MACGREGOR, J. (2003). Students' percepcions of quality in higher education. Quality Assurance in Educacion, 11(1), 15-20.

KEMBER, D. (2004). Interpreting student workload and the factors which shape students' perceptions of their workload. tudies in Higher Education, 29(2), 165184.

KEMBER, D., y LEUNG, D. (1998). Influences upon students' perceptions of workload. Educational Psychology, 18(3), 293-307.

KEMBER, D., y LEUNG, D. Y. P. (2005a). The influence of active learning experiences on the development of graduate capabilities. Studies in Higher Education, 30, 157-172.

KEMBER, D., y LEUNG, D. Y. P. (2005b). The influence of the teaching and learning environment on the development of generic capabilities needed for a knowledgebased society. Learning Environments Research, 8, 245-266.

KEMBER, D., y LEUNG, D. Y. P. (2006). Characterising a teaching and learning environment conducive to making demands on students while not making their workload excessive. Studies in Higher Education, 31(2), 185-198.

KEMBER, D., NG, S., TSE, H., WONG, E. T. T., Y POMFRET, M. (1996). An examination of the interrelationships between workload, study time, learning approachers and academic outcomes. Studies in Higher Education, 21(3), 347-358. 
LAWLESS, C. (2000). Using learning activities in Mathematics: workload and study time. Studies in Higher Education, 25(1), 97-111.

LEUNG, D. Y. P., Y KEMBER, D. (2005). The influence of the part-time study experience on the development of generic capabilities. Journal of Further and Higher Education, 29, 91-101.

LEUNG, D. Y. P., Y KEMBER, D. (2006). The influence of teaching approach and teacher-student interaction on the development of graduate capabilities. Structural Equation Modelling, 13, 164-186.

LIZZIO, A., WILSON, K., Y SIMONS, R. (2002). University students' perceptions of the learning environment and academic outcomes: implications for theory and practice. Studies in Higher Education, 27(1), 27-52.

LOCKWOOD, F. (1992). Activities in self-instructional texts. London: Kogan Page.

MARTÍNEZ-COCÓ, B., GARCÍA, J. N., ROBLEDO, P., DÍEZ, C., ÁlVAREZ, M. L., MARBÁN, J. M., DE CASO, A. M., FIDALGO, R., ARIAS-GUNDÍN, O., PACHECO, D. I., Y RODRÍGUEZ, C. (2008). Valoración Docente de las Metodologías Activas: un Aspecto Clave en el Proceso de Convergencia Europea. Aula Abierta, 35(1-2), 35-48.

MARTON, F., HOUNSELL, D., Y ENTWISTLE, N. (EDS.). (1984). The experience of learning. Edinburgh: Scottish Academic Press.

MATEOS, V. L. Y MONTANERO, M. (EDS.) (2008). Diseño e implantación de Títulos de Grado en el Espacio Europeo de Educación Superior. Madrid: Editorial Narcea.

MINISTERIO DE EDUCACIÓN CULTURA Y DEPORTE (2003). La integración del sistema universitario español en el espacio europeo de enseñanza superior. Documento-Marco. Madrid: Ministerio de Educación, Cultura y Deporte.

MORGAN, A. R., TAYLOR, E., Y GIBBS, G. (1982). Variations in students' approaches to studying. British Journal of Educational Technology, 13(2), 107113.

PARAHOO, K. (1999). Nursing research: principles, problems and issues. London: Macmillan.

PARKINSON, T.J., GILLING, M., Y SUDDABY, G.T. (2006). Workload, study methods, and motivation of students within a BVSc program. Journal of Veterinary Medical Education, 33(2), 253-265.

REISSLEIN, M., TYLAVSKY, D. J., MATAR, B., SEELING, P., Y REISSLEIN, J. (2007). Active and cooperative learning in a freshman digital design course: impact on persistence in engineering and student motivational orientation. En Proceedings of the 37th ASEE/IEEE frontiers in education conference. . Milwauky, USA.

REYES, J. R., VALDÉS, A. Y CASTAÑO, S. (2006). Prácticum y carga de trabajo. Revista de Investigación Educativa, 24(2), 557-574. 
RYAN, M.T., IRWIN, J.A., BANNON, F.J., MULHOLLAND, C.W., Y BAIRD, A.W. (2004). Observations of veterinary medicine students' approaches to study in pre-clinical years. Journal of Veterinary Medical Education, 31(3), 242-254.

SPRONKEN-SMITH, R. (2005). Implementing a problem-based learning approach for teaching research methods in Geography. Journal of Geography in Higher Education, 29(2), 203-221.

STIGGINS, R. (2006). Assessment for learning: A key to motivation and achievement. EDge, 2(2), 3-19.

SUTTON, R. (2007). Veterinary students and their reported academic and personal experiences during the first year of veterinary school. Journal of Veterinary Medical Education, 34(5), 645-651.

WOODLEY, A., Y PARLETT, M. (1983). Student drop-out. Teaching at a Distance, 24, 2-23.

ZABALA, A. Y ARNAU, L. (2008). Once ideas clave: cómo aprender y enseñar competencias ( $2^{\mathrm{a}}$ ed.). Barcelona: Graó.

ZURIFF, G. E. (2003). A method for measuring student study time and preliminary results. Student College Journal, 37, 72-78.

\section{Correspondencia con el autor:}

Carles ROCA-CUBERES

Universitat Pompeu Fabra, Departamento de Comunicación

Roc Boronat 138, 08018 Barcelona

email: carles.roca@upf.edu 


\title{
Anexo 1: cuestionario dedicación temporal del estudiante
}

\author{
ASIGNATURA: \\ CÓDIGO DE LA ASIGNATURA:
}

1. Cuantifica en tiempo tu asistencia, durante esta semana, para esta asignatura a:

MINUTOS

Clases en grupo grande (magistral)

Seminarios

Tutorías

2. Marca cuáles de les siguientes actividades has hecho durante esta semana para esta asignatura, y cuantifica el tiempo dedicado a:

\section{MINUTOS}

$\square$ Ejercicios de redacción

$\square$ Lecturas de artículos, textos, libros

$\square$ Comentarios de texto

$\square$ Ejercicios de lengua (de gramática, vocabulario,...)

$\square$ Ejercicios de traducción

$\square$ Impresión de documentos (apuntes, artículos, PowerPoints,...)

$\square$ Revisión de textos ya corregidos

$\square$ Pasar los apuntes a limpio

$\square$ Hacer resúmenes o esquemas de artículos, textos, libros

$\square$ Buscar información en Internet

$\square$ Buscar información en la bibliografía recomendada

$\square$ Estudiar para exámenes

$\square$ Preparar exposiciones orales individuales

$\square$ Encontrarme con mi grupo para la preparación de un trabajo

$\square$ Consultar documentos audiovisuales

$\checkmark$ Otras (indicar cuáles) 
Otras (indicar cuáles)

\section{Anexo 2: cuestionario sobre la adquisición y evaluación de competencias}

\section{ASIGNATURA:}

\section{CÓDIGO DE LA ASIGNATURA:}

1. Hay diferencias entre lo que se plantea en las competencias de esta asignatura y lo que has aprendido?

Cuáles son esas diferencias?

2. Te han evaluado de las competencias propuestas en la guía docente? 